\title{
Editorial ESB-ITA 2017 special issue
}

Anna Pandolfi ${ }^{1}$, Giuseppe Vairo ${ }^{2}$

${ }^{1}$ Department of Civil and Environmental Engineering, Politecnico di Milano (Polytechnic University of Milan), Milan, Italy; ${ }^{2}$ Department of Civil Engineering and Computer Science, Università degli Studi di Roma "Tor Vergata", Rome Italy

This special issue of the Journal for Modeling in Ophthalmology collects, in the form of extended abstracts, contributions presented during the Thematic Symposium on Eye Biomechanics, organized within the VII Annual Meeting of the Italian Chapter of the European Society of Biomechanics (ESB-ITA 2017) held on September 28-29, 2017 in Rome, Italy. The scientific and administrative organization of the general meeting was committed to the Università degli Studi di Roma "Tor Vergata", while the scientific coordination of the thematic symposium was assigned to the Politecnico di Milano.

The thematic symposium addressed the behavior of heathy and diseased eyes involving diverse areas of physics such as solid mechanics, fluid mechanics, and electro-chemistry. The focus was on modeling the complex physics of the eye, regarded as a fundamental point to improve patient outcomes in current clinical practice. Clinical experiences in ophthalmology, consistent theoretical formulations, and advanced computational modeling methods have been presented as the results of a successful cooperation between clinicians, engineers, and physicists.

In recognition of the high quality of the thematic symposium, approaching topics at the cutting edge of research in ocular biomechanics, this issue collects posters and oral presentations. Contributions describe computational models for the analysis of ocular biomechanics, useful for simulating, planning and optimizing surgical procedures (Sala et al. and Ariza-Gracia et al.); theoretical models and experimental strategies for the investigation of the biomechanical etiology of eye diseases, e.g., glaucoma, age-related macular degeneration, vitreoschisis, myopia, and diabetic retinopathy (Stefanoni et al., Sacco et al., and Salerni et al.), as well as for the optimization of possible therapeutic strategies (Ferroni et al. and Davvalo Khongar et al.); experimental studies for the identification of the mechanical properties of the tissues (Zhoula et al., Kazaili and Akhtar, and Lipari et al.) and the optimization of surgical procedures (Romano et al., Nepita et al., and Stocchino et al.); constitutive models of ocular tissues (Vasta et al. and Recrosi et al.); and computational approaches for the simulation of mechanical-based diagnostic procedures (Montanino et al. and Ariza-Gracia et al.).

Correspondence: Anna Pandolfi, Department of Civil and Environmental Engineering, Politecnico di Milano, Piazza Leonardo da Vinci 32, 20133 Milano, Italy.

E-mail: anna.pandolfi@polimi.it 
Although the summaries collected here refer to selected front-line studies developed mainly within the Italian community, all contributions testify explicitly that the efforts arising from the Italian scientists in the field of ocular biomechanics are strongly intertwined with the international research network, and that Italian research plays a major role in the international scene. 